ULTIMA DÉCADA No25, CIDPA VALPARAísO, DICIEMBRE 2006, PP. 43-64.

\title{
PROYECTOS FAMILIARES Y DE PAREJA ENTRE LOS JOVENES DE SANTIAGO DE CHILE
}

\author{
MARÍA SOLEDAD HERRERA PONCE*
}

\begin{abstract}
RESUMEN
Los jóvenes chilenos valoran altamente y se perciben en el futuro viviendo en familia, constituida con hijos y generalmente casados. En este artículo se profundiza en las ideas y proyectos que tienen los jóvenes acerca de la familia. Se destaca la prioridad que tienen los hijos en la conformación de la familia, tanto a nivel de las percepciones como de proyectos. El matrimonio tiene un lugar secundario pero no por ello de poca importancia; la mayoría de los jóvenes se proyecta viviendo en matrimonio en el futuro, aunque su modo de entrada será cada vez más a través de una convivencia previa. Los datos provienen principalmente de una encuesta representativa de los jóvenes entre 15 y 30 años, realizada en Santiago de Chile durante el año 2004 y financiada por la Fundación Andes, que tenía como propósito estudiar las experiencias, opiniones y proyectos familiares de los jóvenes, los cuales se ilustran también con citas textuales de jóvenes entrevistados en profundidad o que participaron en grupos focales. Adicionalmente, en cada apartado se complementan los resultados de dicha encuesta con datos que permitan contextualizar los hallazgos de la investigación, utilizando otras fuentes estadísticas, principalmente los Censos de población chilena de 1992 y 2002, y estadísticas internacionales de Naciones Unidas.
\end{abstract}

Palabras clave: Cambio familiar en Chile, Estructuras de hogar, REPRESENTACIONES FAMILIARES

* Socióloga, Pontificia Universidad Católica de Chile. Doctora en Sociología, Universidad Autónoma de Madrid. Profesora del Instituto de Sociología de la Pontificia Universidad Católica de Chile, Santiago, Chile. E-Mail: mherrepo@uc.cl. 


\section{RESUMO}

\section{PROJETOS FAMILIARES E DE CASAL ENTRE OS JOVENS DE SANTIAGO DO CHILE}

Os jovens chilenos valorizam muito e se percebem no futuro vivendo em família constituída com filhos e geralmente casados. Neste artigo as idéias e os projetos dos jovens sobre família são aprofundados. Destaca-se a prioridade dos filhos na conformação da família, tanto no nível das percepções como dos projetos. O casamento tem lugar secundário, mas não de pouca importância; a maioria dos jovens tem projeto de casamento para o futuro, ainda que seu modo de entrada no casamento seja cada mais vez através de convivência prévia. Os dados provêm principalmente de uma pesquisa representativa de jovens entre 15 e 30 anos, realizada em Santiago durante ano de 2004, que tinha como objetivo estudar as experiências, opiniões e projetos familiares dos jovens, ilustrados também com falas textuais dos jovens entrevistados em profundidade, ou que participaram em grupos focais. Adicionalmente, em cada parte, completam-se os resultados desta pesquisa com dados que permitem contextualizar as descobertas da investigação, utilizando outras fontes estatísticas, principalmente os censos de população chilenos de 1992 e 2002, bem como estatísticas internacionais da Organização das Nações Unidas.

Palavras chave: Mudanças familiares no Chile, Estruturas domÉsticas, RePRESENTAÇÕES FAMILIARES

\section{Abstract}

\section{FAMILY AND COUPLE PROJECTS AMONG THE YOUNG OF SANTIAGO DE CHILE}

Family life is highly valued among Chilean young people. They visualize their future living in family with children and generally married. This article explores young people's ideas and projects regarding the family. It highlights the prioriy of children in the making of a family as a perception as well as a project. Marriage is in a secondary place, however, it is not less important; most of the young persons visualize themselves married in a future, but each time more often, through a previous period of living together. The information comes mainly from a representative enquiry of youngsters between 15 and 30 years old, carried out in Santiago during year 2004 with the purpose of studying the experiences, opinion and family projects of the young people. The information is also enlighted with textual quotes from youngsters interviewed thoroughly or that joined focal groups. In addition, in each point, the results of such enquiry are complemented with data which allows to put in perspective the foundings of the investigation using other statistical information mainly the Chilean Population Census of year 1992 and year 2002, as well as United Nations international statistics too.

Key Words: FAmily ChangeS IN CHILE, Home STRUCtures,

FAMILY REPRESENTATIONS 


\section{INTRODUCCIÓN}

EN CHILE, EN CONCORDANCIA con lo que ha estado ocurriendo a nivel mundial, se ha estado produciendo una mayor diversificación de las estructuras familiares, lo que ha ido acompañado de un cambio en las percepciones acerca de lo que se considera realmente una «familia». Ha ido variando el concepto de familia, abriéndose a otras formas distintas a lo que se entiende como «familia tradicional», compuesta por padre, madre e hijos. Cabe señalar, no obstante que en los distintos países tradicionalmente se ha agregado otro tipo de personas a esta familia nuclear, siendo en América Latina la familia extensa (con presencia de otros familiares además del núcleo principal) el patrón de lo que se entiende usualmente como familia tradicional.

Si bien la mayoría de los hogares chilenos continúa siendo una pareja con hijos (con o sin otras personas adicionales), este tipo de hogares ha disminuido desde un 58\% en el año 1992 a un 52\% en el año 2002. Como contraparte, han aumentado los hogares unipersonales y las parejas sin hijos, especialmente por efecto del mayor envejecimiento de la población, donde este tipo de hogares son más frecuentes (Gubbins, Browne y Bagnara, 2004). El mejoramiento de las condiciones generales de vida de la población chilena durante la década de los noventa (Valenzuela y Herrera, 2003), ha significado un aumento de las personas que tienen acceso a una vivienda propia, lo que ha tenido como consecuencia un incremento del porcentaje de personas que encabeza - ya sea como jefe o pareja - su propio hogar. Sin embargo, entre los jóvenes ha disminuido la jefatura de hogar, debido a que cuentan con mayores oportunidades educativas, lo que los mantiene por más tiempo en casa de sus padres. Entre los jóvenes que se han independizado de los padres, llama la atención el hecho que cada vez es menos para constituir una familia formada por una pareja con hijos, aumentando los que se van a vivir solos o únicamente con sus parejas, ${ }^{1}$ lo que puede estar relacionado con el aplazamiento en la edad en que se tienen hijos.

Hasta ahora se han analizado las estructuras familiares donde viven los jóvenes, pero, ¿constituyen realmente «familias»?, ¿cuáles son los límites de lo que se considera una familia?, ¿cómo son las relaciones que establecen los jóvenes con los miembros de sus familias?, ¿qué proyectos tienen respecto a formar sus propias familias? Estas

1 Censos chilenos de 1992 y 2002, tabulaciones propias. 
son algunas de las preguntas que se intentarán responder, vale decir, ¿qué modelo de familia está presente en la percepción de los jóvenes?

Está claro que lo primero que define la idea de familia entre los jóvenes es la presencia de hijos, puesto que aunque no esté presente una pareja, la mayoría opina que hay familia en el caso de «madre o padre solos con hijos» ( $84 \%$ y $82 \%$, respectivamente) (Tabla 1). En la definición de una familia de parte de los jóvenes, el matrimonio legal resulta menos importante que la presencia de hijos: el nivel de acuerdo con que una «pareja que convive con hijos» es una familia $(92 \%)$ es sólo levemente inferior a la aceptación de la idea convencional de familia constituida por un «matrimonio con hijos» (98\%). La mayor importancia de los hijos como constitutivos de una familia queda de manifiesto en el hecho de que un «matrimonio solo, sin hijos» resulta incluso menos aceptado que la pareja que convive con hijos (79\%).

Al profundizar en las ideas de familia que tienen los jóvenes, además de la importancia de los hijos, resalta la persistencia del sentimiento amoroso como fundamento de éstas. En este contexto, el matrimonio se legitima no por el contrato legal o por el sacramento religioso, sino por la presencia de hijos y por la continuidad del amor existente tanto entre los cónyuges como en la relación con los hijos:

[Yo pienso] que pareja es pareja y al tener un hijo ya es una familia [...] por eso cuando se casan dicen vamos a formar una familia, porque se refiere a los hijos que van a tener. ${ }^{2}$

Porque igual casarse es como firmar un papel y que digan textualmente que estai casado, pero si tú sentís que estai casado con una persona, estai casado poh [o sea] depende de todo, de cómo se llevan juntos. ${ }^{3}$

La familia se forma con el hijo; en el momento en que dejamos de ser pareja y somos una familia es el momento en que somos tres y no dos. ${ }^{4}$

En este sentido, un padre que no es el padre genético puede ser considerado más como parte de la familia que el verdadero padre cuando no establece una relación afectiva con los hijos (en el sentido de amor y preocupación), incluso si aporta económicamente:

2 Grupo focal, liceo, nivel socioeconómico medio bajo.

3 Grupo focal, colegio, nivel socioeconómico medio alto.

4 Grupo focal, universidad, nivel socioeconómico medio alto. 
Yo encuentro que el término de una familia no lo hace un papel y una ceremonia, lo hace un sentimiento [la pareja de una madre sola] si se lleva bien con el hijo si [es parte de la familia], pero si no los pescan y chao no [y si la pareja no pesca pero aporta solamente la parte económica] sería como cualquier persona que te diera plata. ${ }^{5}$

Sin embargo, si se suma la cohabitación y el hecho de no tener hijos, esta situación es menos aceptada como familia. En otras palabras, la convivencia sin casarse es bastante menos apreciada como familia cuando no se tienen hijos (57\%), en comparación con el matrimonio sin hijos, probablemente porque se supone que este último incorporará hijos en un futuro próximo, mientras que la cohabitación puede ser vivenciada sólo como una etapa de "prueba», que no necesariamente tendrá la estabilidad de un matrimonio.

No obstante lo anterior, cabe señalar que una importante proporción de jóvenes efectivamente considera como familia a las parejas convivientes sin hijos, en cuanto exista el fundamento del amor que, como se señaló anteriormente, es considerado como uno de los elementos constitutivos de las familias:

[Una pareja que convive sin hijos] sí son familia [...] si se aman y todo yo creo que sí son familia porque yo creo que eso es lo que cuenta. ${ }^{6}$

En referencia a las parejas del mismo sexo, éstas son aceptadas como familias casi por la mitad de los encuestados cuando hay hijos, mientras que cuando no los hay, sólo son admitidas por un 30\% de los jóvenes. La aceptación de este tipo de familias es bastante menor que en otros países europeos, por ejemplo, en Madrid un 76\% de los jóvenes considera como familia a las parejas del mismo sexo con hijos y un $53 \%$ a este tipo de parejas pero sin hijos (Meil, 2005).

Los resultados anteriormente señalados no varían significativamente por sexo, pero sí lo hacen por edad y nivel socioeconómico, de la siguiente manera: i) las parejas convivientes y los padres solteros son más aceptados a mayor edad, es decir, con el paso del tiempo los jóvenes aumentan sus niveles de tolerancia, lo que puede ser producto de que conocen más de cerca otras realidades distintas a las de sus familias, pero también puede ser una justificación posterior a la adopción de este tipo de conductas; ii) la presencia de hijos suele ser más

5 Grupo focal, colegio, nivel socioeconómico medio alto.

6 Grupo focal, colegio, nivel socioeconómico medio alto. 
valorada para limitar el concepto de familia a mayor nivel socioeconómico (Tabla 1).

Tabla 1

Ideas de familia según edad y nivel socioeconómico (en porcentajes)

\begin{tabular}{|l|c|c|c|c|c|c|c|}
\hline \multirow{2}{*}{ Es una familia... } & \multicolumn{3}{|c|}{ Edad } & \multicolumn{3}{c|}{ Nivel socioeconómico } & \multirow{2}{*}{ Total } \\
\cline { 2 - 7 } & $\mathbf{1 5 - 1 8}$ & $\mathbf{1 9 - 2 4}$ & $\mathbf{2 5 - 3 0}$ & Bajo & Medio & Alto & \\
\hline Matrimonio con al menos un hijo & 96,9 & 97,3 & 98,3 & 97,9 & $95,8^{3}$ & 99,3 & 97,6 \\
\hline $\begin{array}{l}\text { Pareja que convive sin casarse } \\
\text { con al menos un hijo }\end{array}$ & 86,3 & $92,0^{1}$ & $96,0^{1}$ & 92,5 & 91,0 & 92,7 & 92,1 \\
\hline Madre sola con al menos un hijo & 75,3 & $84,0^{1}$ & $89,6^{1}$ & 83,0 & $81,2^{3}$ & 88,3 & 83,9 \\
\hline Padre solo con al menos un hijo & 75,0 & $82,5^{1}$ & $86,5^{1}$ & 80,8 & $79,4^{3}$ & 87,0 & 82,1 \\
\hline Matrimonio sin hijos & 74,2 & 80,8 & 79,0 & $71,7^{3}$ & $78,2^{3}$ & 88,2 & 78,5 \\
\hline $\begin{array}{l}\text { Pareja que convive sin casarse y } \\
\text { sin hijos }\end{array}$ & 45,0 & $59,9^{1}$ & $61,3^{1}$ & 55,9 & 54,0 & 60,9 & 56,7 \\
\hline $\begin{array}{l}\text { Pareja del mismo sexo con al } \\
\text { menos un hijo }\end{array}$ & $53,6^{2}$ & $51,4^{2}$ & 41,4 & $45,4^{3}$ & 46,0 & 54,6 & 48,2 \\
\hline Pareja del mismo sexo sin hijos & 29,6 & 34,5 & 27,3 & $27,4^{3}$ & 29,2 & 36,4 & 30,5 \\
\hline & $\mathrm{N}=310$ & $\mathrm{~N}=449$ & $\mathrm{~N}=457$ & $\mathrm{~N}=474$ & $\mathrm{~N}=401$ & $\mathrm{~N}=340$ & $\mathrm{~N}=1216$ \\
\hline
\end{tabular}

Pregunta: «Desde tu punto de vista, de las siguientes formas de vida, ¿cuáles consideras que son una familia?» (respuestas: «sí» o «no»).

1 Prueba de comparación de proporciones respecto de «15-18 años» es significativa al nivel de 0,05 (prueba bilateral).

2 Prueba de comparación de proporciones respecto de «25-30 años» es significativa al nivel de 0,05 (prueba bilateral).

3 Prueba de comparación de proporciones respecto de «nivel socioeconómico alto» es significativa al nivel de 0,05 (prueba bilateral).

El nivel de satisfacción con las relaciones que los jóvenes establecen con sus familias es medianamente alto, pero generalmente inferior a la evaluación que hacen de las relaciones con sus amigos (Tabla 2). Un $41 \%$ responde que la familia en que creció constituye totalmente un modelo a seguir, pero prácticamente un quinto de los jóvenes siente que su familia de origen no es un ejemplo. La calidad de las relaciones familiares está fuertemente determinada por el tipo de relación de pareja que han tenido sus padres, de manera que cuando éstos no han vivido juntos se evalúa de peor manera tanto a la familia en general, como las relaciones con sus madres, con sus padres, sus hermanos y primos paternos.

Como es esperable, donde se producen las mayores diferencias es en la calificación de las relaciones entre los padres. Cuando éstos no viven juntos, son muy mal evaluados, en comparación con cuando se encuentran viviendo como pareja. Cabe destacar que no existen diferencias en la evaluación de las relaciones entre los padres según si 
ellos están legalmente casados o si están cohabitando sin casarse (Tabla 2). ${ }^{7}$ Esto es concordante con la relativa alta aceptación de la convivencia entre los jóvenes, ya que al menos en la calidad de las relaciones entre ambos miembros de una pareja ellos no ven mucha diferencia.

Tabla 2

Evaluación familiar según situación conyugal de los padres

\begin{tabular}{|ll|c|c|c|c|}
\hline \multirow{2}{*}{} & \multicolumn{4}{|c|}{ Situación conyugal padres } \\
\cline { 3 - 6 } & Casados & $\begin{array}{c}\text { Convi- } \\
\text { ven }\end{array}$ & $\begin{array}{c}\text { No viven } \\
\text { juntos }\end{array}$ & Total \\
\hline Constituye un modelo a seguir la familia en que creció & $5.0^{1}$ & $4.9^{1}$ & 4.2 & 4.7 \\
[escala de 1 (no, en absoluto) a 6 (sí, totalmente)]. & & $5.4^{1}$ & $5.4^{1}$ & 4.9 & 5.2 \\
\hline Qué tan satisfactorias considera & Su madre & $4.9^{1}$ & $4.6^{1}$ & 3.7 & 4.6 \\
las relaciones que mantiene & Su padre & $5.1^{1}$ & 4.9 & 4.8 & 4.9 \\
actualmente con... & Hermano(s) & 4.7 & 4.5 & 4.5 & 4.6 \\
[escala de 1 (nada satisfactorias) a a Abuelos maternos & Abuelos paternos & 4.0 & 4.0 & 3.7 & 3.9 \\
6 (muy satisfactorias)]. & Primos maternos & 4.4 & 4.3 & 4.3 & 4.3 \\
& Primos paternos & $3.8^{1}$ & 3.5 & 3.5 & 3.7 \\
& Amigos hombres & 5.3 & 4.8 & 5.2 & 5.3 \\
& Amigas mujeres & 5.2 & 5.3 & 5.2 & 5.2 \\
\hline Calificación de la relación que & En la actualidad & $5.0^{1}$ & $4.9^{1}$ & 2.8 & 4.5 \\
matienen sus padres entre sí & En el pasado & & & & \\
[escala de 1 (nada satisfactorias) a a & & $4.7^{1}$ & $4.5^{1}$ & 3.6 & 4.3 \\
6 (muy satisfactorias)]. & & & & \\
\hline \multicolumn{2}{|r|}{} \\
\hline
\end{tabular}

Se excluyen los casos que no conocieron a cada tipo de persona.

1 Prueba de comparación de proporciones respecto de «no viven juntos» es significativa al nivel de 0,05 (prueba bilateral).

La importancia de la familia es algo generalizado entre los jóvenes; la mayoría considera como «bastante» o «extremadamente» importante para el futuro tanto vivir en pareja como tener hijos y llevarse bien con sus familiares. Esto último es lo más valorado por los jóvenes, especialmente llevarse bien con sus hijos, lo que es considerado incluso como más importante que haber tenido hijos. El vivir en pareja es casi igualmente valorado que casarse, y ambos son menos importantes que tener hijos. Estos resultados son consistentes con lo mencionado anteriormente, acerca de que los hijos son más importantes que la pareja en la constitución de las familias de los jóvenes. Todas estas valoraciones son mayores entre los de 25 y 30 años $^{8}$ (Tabla 3 ).

$7 \quad$ Las diferencias por sexo, edad y nivel socioeconómico no son importantes.

8 No se observan diferencias significativas respecto de sexo y nivel socioeconómico. 
Tabla 3

Importancia de las relaciones familiares a futuro según edad (en porcentajes)

\begin{tabular}{|c|c|c|c|c|}
\hline \multirow{2}{*}{$\begin{array}{l}\text { Extremadamente (bastante) importante } \\
\text { en el futuro... }\end{array}$} & \multicolumn{3}{|c|}{ Edad } & \multirow[t]{2}{*}{ Total } \\
\hline & $15-18$ & $19-24$ & $25-30$ & \\
\hline Vivir en pareja & $22,2^{1}(59.5)$ & $26,7^{1}(57.3)$ & $34,2(52.3)$ & $28,4(56.0)$ \\
\hline Haber tenido hijos & $33,9^{1}(55.2)$ & $41,5^{1}(47.7)$ & $57,6(38.7)$ & $45,6(46.2)$ \\
\hline Estar casado/a & $22,2(45.8)$ & $21,5^{1}(41.5)$ & $29,4(42.2)$ & $24,6(42.9)$ \\
\hline Llevarse bien con sus padres & $53,3(43.8)$ & $47,4^{1}(48.2)$ & $57,9(36.9)$ & $52,9(42.8)$ \\
\hline Llevarse bien con sus hermanos & $48,6(45.6)$ & $46,5^{1}(47.9)$ & $56,4(39.0)$ & $50,8(44.0)$ \\
\hline Llevarse bien con los hijos & $75,9(23.5)$ & $73,6^{1}(25.2)$ & $83,0(16.1)$ & $77,7(21.4)$ \\
\hline Vivir cerca de sus padres y/o hermanos & $15,9(46.6)$ & $14,5(41.5)$ & $18,7(47.4)$ & $16,4(45.0)$ \\
\hline & $\mathrm{N}=310$ & $\mathrm{~N}=449$ & $\mathrm{~N}=457$ & $\mathrm{~N}=1216$ \\
\hline
\end{tabular}

Respecto de las expectativas acerca de cómo se ven los jóvenes en el futuro, cabe resaltar que la alta valoración de los hijos es consistente con las expectativas que tienen los jóvenes de tener hijos en el futuro, donde la mayoría cree que en el futuro tendrá (más) hijos $(85 \%)$. Sin embargo, la valoración de la cohabitación - que resulta sólo levemente inferior al matrimonio- no es coherente con las propias expectativas de los jóvenes, ya que mientras un $78 \%$ se ve como casado, sólo un $38 \%$ se percibe como conviviendo sin casarse. Esto puede estar indicando que la convivencia juvenil no se espera como un estado permanente en la vida de los jóvenes, sino que es más bien una etapa previa al matrimonio, especialmente cuando no se tienen hijos. Cabe destacar que un $16 \%$ de los jóvenes piensa que estará separado, anulado o divorciado en el futuro, lo que demuestra una desconfianza frente al posible éxito en sus relaciones de pareja. También cabe agregar que un cuarto de los jóvenes cree que estará viviendo solo (Tabla 4).

Ahora cabe preguntarse quiénes son los que tienen menores expectativas de estar viviendo en un tipo de familia convencional (matrimonio con hijos) en el futuro. Se podría pensar que la situación conyugal de los padres sería el factor más determinante, por ejemplo, que los jóvenes vean como normal convivir antes que casarse; también hay investigaciones que han mostrado que la experiencia de separación de los padres influye en este mismo tipo de vivencias entre sus hijos (Wellings y Wadsworth, 1990; Wolfinger, 2000). La Encuesta Nacional de Familia (DESUC, 1998) dio cuenta de que los hijos de 
padres separados tienden a tener una actitud más favorable hacia el divorcio; sin embargo, en la encuesta de jóvenes realizada en Santiago no se ha encontrado dicha relación. Por otra parte, si bien se encuentra una relación estadísticamente significativa entre las expectativas de estar separados y vivencia de separación de los padres, esta relación deja de ser significativa al controlar por nivel socioeconómico (Tabla 5). Los jóvenes con padres separados tienen mayores expectativas de fracasar en sus relaciones de pareja no porque hayan experimentado este tipo de experiencias, sino porque viven en condiciones económicas más desfavorables, donde se da mayor inestabilidad conyugal.

La relación entre nivel socioeconómico bajo y mayores probabilidades de divorcio ha sido comprobada en varios estudios internacionales. Ésta se da a través de dos mecanismos: i) por una parte, las personas de menor nivel económico tienen menor acceso a la educación, que puede proveer de mayores recursos sociales para resolver conflictos (Heaton, 2002; Orbuch y otros, 2002); ii) por otra parte, la falta de ingresos genera estrés en las relaciones interpersonales (Kiernan y Mueller, 1990), lo que puede ser fuente de inestabilidad.

Por el contrario, la experiencia de cohabitación de los padres sí tiene alguna influencia independiente sobre las expectativas de cohabitar de los hijos, como se desprende del resultado de la regresión logística expuesta en la Tabla 6. Además, los efectos se suman, de manera que el hecho de provenir de familia con padres convivientes y pertenecer a un nivel socioeconómico más bajo aumentan las probabilidades de proyectarse en el futuro formando una pareja conviviente sin casarse (Tabla 5).

Tabla 4

Expectativas de vida en familia en el futuro según sexo y nivel socioeconómico

(en porcentajes)

\begin{tabular}{|l|c|c|c|c|c|c|}
\hline \multirow{2}{*}{$\begin{array}{l}\text { En 25 años, cree que con toda seguridad } \\
\text { o probablemente sí... }\end{array}$} & \multicolumn{2}{|c|}{ Sexo } & \multicolumn{3}{c|}{ Nivel socioeconómico } & \multirow{2}{*}{ Total } \\
\cline { 2 - 7 } & Hombre & Mujer & Bajo & Medio & Alto & \\
\hline Estará viviendo solo/a & 21,7 & 27,8 & $29,2^{2}$ & $25,1^{2}$ & 19,0 & 25,0 \\
\hline Tendrá (más) hijos & 90,1 & 80,9 & $82,9^{2}$ & $81,5^{2}$ & 93,1 & 85,2 \\
\hline $\begin{array}{l}\text { Estará separado/a, anulado/a o divorcia- } \\
\text { do/a }\end{array}$ & 13,8 & 18,5 & $22,5^{2}$ & $18,5^{2}$ & 5,1 & 16,3 \\
\hline Estará conviviendo sin casar & 43,2 & 33,9 & $46,0^{2}$ & $38,6^{2}$ & 27,0 & 38,3 \\
\hline Estará casado/a & 80,8 & 75,0 & $70,1^{2}$ & $78,8^{2}$ & 86,9 & 77,7 \\
\hline
\end{tabular}


(chi cuadrado).

2 Prueba de comparación de proporciones respecto de «25-30 años» es significativa al nivel de 0,05 (prueba bilateral).

Tabla 5

Expectativas de vida en familia en el futuro según nivel socioeconómico y situación conyugal de los padres (en porcentajes)

\begin{tabular}{|c|c|c|c|c|c|c|c|c|c|c|c|c|}
\hline & \multicolumn{2}{|c|}{ Clase alta } & \multicolumn{2}{|c|}{ Clase media } & \multicolumn{3}{|c|}{ Clase baja } & \multicolumn{4}{|c|}{ Total } \\
\hline & & $\begin{array}{c}\text { Padres } \\
\text { casados }\end{array}$ & $\begin{array}{c}\text { Padres } \\
\text { no } \\
\text { viven } \\
\text { juntos }\end{array}$ & $\begin{array}{l}\text { Padres } \\
\text { casados }\end{array}$ & $\begin{array}{c}\text { Padres } \\
\text { no } \\
\text { viven } \\
\text { juntos }\end{array}$ & $\begin{array}{c}\text { Padres } \\
\text { casados }\end{array}$ & $\begin{array}{l}\text { Convi- } \\
\text { ven }\end{array}$ & $\begin{array}{c}\text { No } \\
\text { viven } \\
\text { juntos }\end{array}$ & $\begin{array}{l}\text { Padres } \\
\text { casados }\end{array}$ & $\begin{array}{l}\text { Padres } \\
\text { convi- } \\
\text { ven }\end{array}$ & $\begin{array}{c}\text { Padres } \\
\text { no } \\
\text { viven } \\
\text { juntos }\end{array}$ & Total \\
\hline \multirow{3}{*}{\begin{tabular}{|l} 
Estará \\
separado, \\
anulado o \\
divorcia- \\
do \\
\end{tabular}} & No & 95,3 & 94,0 & 84,8 & 77,7 & 79,5 & 78,6 & 75,3 & 86,3 & 78,3 & 80,8 & 83,8 \\
\hline & Sí & 4,7 & 6,0 & 15,2 & 22,3 & 20,5 & 21,4 & 24,7 & 13,7 & 21,7 & 19,2 & 16,2 \\
\hline & $\mathrm{n}$ & 191 & 106 & 219 & 126 & 208 & 33 & 181 & 617 & 62 & 414 & 1093 \\
\hline \multirow{3}{*}{\begin{tabular}{|l|} 
Estará \\
convi- \\
viendo sin \\
casarse
\end{tabular}} & No & 73,9 & 71,7 & 64,9 & 57,5 & 59,9 & 37,6 & 50,4 & 66,0 & 44,9 & 58,0 & 61,8 \\
\hline & Sí & 26,1 & 28,3 & 35,1 & 42,5 & 40,1 & 62,4 & 49,6 & 34,0 & $55,1^{1}$ & 42,0 & 38,2 \\
\hline & $\mathrm{n}$ & 199 & 111 & 227 & 132 & 218 & 34 & 190 & 643 & 63 & 433 & 1139 \\
\hline
\end{tabular}

Nota: se eliminaron columna con un tamaño muestral inferior a 30 casos.

1 Prueba de comparación de proporciones respecto de «padres casados» es significativa al nivel de 0,05 (prueba bilateral). 
Tabla 6

Coeficientes de regresión logística (variable dependiente: "Tiene expectativas de cohabitar» ${ }^{1}$ )

\begin{tabular}{|l|c|}
\hline & $\exp (\mathrm{B})^{2}$ \\
\hline Padres conviven $^{3}$ & 2.078 \\
\hline Padres no viven juntos $^{3}$ & 1.340 \\
\hline Nivel socioeconómico medio $^{4}$ & 1.657 \\
\hline Nivel socioeconómico alto $^{4}$ & 2.159 \\
\hline
\end{tabular}

1 «Cree que seguramente o probablemente se encontrará conviviendo sin casarse en 25 años más» en comparación con los que NO creen esto.

2 Todos los coeficientes son estadísticamente significativos al nivel $\mathrm{p}=0,05$.

3 Categoría de comparación: padres casados.

4 Categoría de comparación: nivel socioeconómico bajo.

\section{VIDA EN PAREJA: MATRIMONIO, COHABITACIÓN Y DISOLUCIÓN CONYUGAL}

Desde el punto de vista de la vida en pareja, se ha estado vivenciando una progresiva desinstitucionalización del matrimonio. Éste se ha ido haciendo menos frecuente entre los chilenos, a la vez que a nivel de percepciones se ha ido equiparando cada vez más a las cohabitaciones heterosexuales sin matrimonio. Sin embargo, hay que señalar que la cohabitación en Chile ha sido relativamente alta durante un largo período de su historia, especialmente a menor nivel socioeconómico, donde no queda tan claro que un aumento de la cohabitación pueda ser adjudicada a un cambio de valores como un aumento de la individualización, lo que sí estaría ocurriendo en otros países europeos (Herrera, 2002).

Respecto de la evolución de los matrimonios en Chile, cabe destacar que la tasa de matrimonio se ha mantenido relativamente estable durante el siglo veinte, disminuyendo únicamente a partir de los años noventa (INE, 1999 y 2003). En comparación con otros países más desarrollados, la disminución de la tasa de matrimonio en Chile ha sido algo más tardía, por esto el año 1994 se ubicaba por encima de Alemania y España, aunque por debajo de Estados Unidos. Sin embargo, el descenso en los matrimonios durante los noventa fue tan abrupto, que en el año 2001, Chile se ubica por debajo de los países antes mencionados (Tabla 7). La disminución de los matrimonios en Chile tiene como contrapartida un aumento tanto de las personas solte- 
ras como de quienes conviven sin casarse, lo cual ocurre especialmente entre los jóvenes (Herrera y Valenzuela, 2006).

Si bien la expansión de las oportunidades educativas en la última década está explicando en gran parte el aumento de los jóvenes solteros, éste no es el único hecho relacionado. En Chile, la maternidad adolescente ha sido y continúa siendo bastante alta, pero antes era más frecuente que las mujeres contrajeran matrimonio para «legitimizar» a sus hijos a través de dicho vínculo, en un contexto donde legalmente se establecían diferencias entre los hijos nacidos «dentro» y «fuera» del matrimonio. La nueva Ley de Filiación elimina dichas diferencias. En el año 2002 es más frecuente que en 1992 el hecho que las madres adolescentes permanezcan solteras (especialmente a mayor nivel socioeconómico) o que establezcan relaciones de cohabitación sin casarse (lo que es más frecuente a menor nivel socioeconómico). ${ }^{9}$ Por lo tanto, es posible atribuir que una parte de los cambios en las tasas de matrimonio puede relacionarse con los cambios en la legislación chilena.

Tabla 7

Tasa bruta de matrimonio, algunos países 1960-2001

\begin{tabular}{|l|ccccccccc|}
\hline Países & $\mathbf{1 9 6 0}$ & $\mathbf{1 9 6 5}$ & $\mathbf{1 9 7 0}$ & $\mathbf{1 9 7 5}$ & $\mathbf{1 9 8 0}$ & $\mathbf{1 9 8 5}$ & $\mathbf{1 9 9 0}$ & $\mathbf{1 9 9 4}$ & $\mathbf{2 0 0 1}$ \\
\hline Argentina & 7,0 & 6,9 & 7,3 & & 5,9 & & & & 3,5 \\
\hline Chile & 7,4 & 7,6 & 7,6 & 7,4 & 7,7 & 7,5 & 7,5 & 6,5 & 4,2 \\
\hline Perú & 4,4 & 3,9 & 3,8 & 2,8 & 2,5 & & & & \\
\hline Alemania & & & & & & & & 5,4 & 4,7 \\
\hline Alemania Occidental & 9,4 & 8,3 & 7,3 & 6,3 & 5,9 & 6 & 6,5 & & \\
\hline Alemania Oriental & 9,7 & 7,6 & 7,7 & 8,4 & 8 & 7,9 & & & \\
\hline España & 7,8 & 7,1 & 7,3 & 7,6 & 5,9 & 5,2 & & 5,0 & 5,1 \\
\hline Italia & 7,7 & 7,7 & 7,4 & 6,7 & 5,7 & 5,2 & & 5,0 & 4,8 \\
\hline Francia & 7,0 & 7,1 & 7,8 & 7,3 & 6,2 & 4,9 & & 4,4 & 4,9 \\
\hline Estados Unidos & 8,4 & 9,3 & 10,5 & 10,0 & 10,5 & 10,1 & 9,8 & 9,1 & 8,2 \\
\hline
\end{tabular}

Fuente: elaboración propia a partir de datos de United Nations (varios años).

Otra forma que puede adquirir la vida en pareja es la de cohabitación ( $\sin$ casarse). Los cohabitantes en Chile aumentaron desde 5,7\% a 9\% entre 1992 y 2002. Ésta se da con bastante mayor frecuencia a menor nivel socioeconómico (Herrera y Valenzuela, 2006). La experiencia de cohabitación antes de casarse alcanzó al 17\%, según datos de la Encuesta Nacional de Familia realizada en Chile en 1998 (DESUC, 1998).

9 Censos chilenos de 1992 y 2002, tabulaciones propias a partir de una muestra aleatoria del 5\% de los respectivos Censos. 
En comparación con otros países, ésta resulta bastante más baja que en Alemania y Estados Unidos, y más alta que en otros países europeos con fuerte influencia de la Iglesia Católica, como son España y Polonia (Tabla 8).

Tabla 8

Porcentaje de personas casadas que convivió antes de casarse en algunos países (algún año entre 1994 y 1998)

(base: personas de 18 o más años)

\begin{tabular}{|l|l|c|c|c|c|c|c|}
\hline \multicolumn{2}{|c|}{} & $\begin{array}{c}\text { Alemania } \\
\text { Occidental }\end{array}$ & $\begin{array}{c}\text { Alemania } \\
\text { Oriental }\end{array}$ & $\begin{array}{c}\text { Estados } \\
\text { Unidos }\end{array}$ & Polonia & España & Chile \\
\hline \multirow{2}{*}{$\begin{array}{l}\text { Convivió } \\
\text { antes de } \\
\text { casarse }\end{array}$} & Sí & $36,6 \%$ & $46,6 \%$ & $29,2 \%$ & $12,3 \%$ & $7,2 \%$ & $17,4 \%$ \\
\cline { 2 - 8 } & No & $63,4 \%$ & $53,4 \%$ & $70,8 \%$ & $87,7 \%$ & $92,8 \%$ & $82,6 \%$ \\
\hline
\end{tabular}

Fuente: para Chile, DESUC, 1998; resto de países, ISSP, 1994.

Más de dos tercios de los jóvenes santiaguinos desea casarse, aunque en diversas modalidades. Poco menos de un tercio de ellos prefiere casarse con una convivencia previa y en un porcentaje similar desean casarse viviendo antes solos; un 15\% señala que le gustaría casarse sin convivir anteriormente; estas dos últimas alternativas son más frecuentes a mayor nivel socioeconómico. Un porcentaje menor de jóvenes expresa su deseo de convivir sin casarse o habiendo vivido antes solo, situaciones que son más mencionadas a menor nivel socioeconómico. También hay algunas diferencias por edad, en el sentido de que los más jóvenes expresan más deseos de vivir solos, lo que se relaciona seguramente con que quieren salir pronto de las casas de sus padres; en contraparte, los mayores esperan más cohabitar con sus parejas sin casarse (Tabla 9). No hay relaciones importantes ni respecto a sexo ni en cuanto a la situación conyugal de los padres, pero sí hay respecto de la religión, en el sentido que los que no son religiosos prefieren en mayor magnitud la cohabitación (Tabla 10).

Entre los que les gustaría casarse, la mayoría preferiría un matrimonio religioso (63\% por la Iglesia Católica y $9 \%$ por otra religión), de manera consistente con el alto porcentaje de jóvenes que se identifica con alguna religión (80\%). De este modo, más de la mitad de los jóvenes que prefiere un matrimonio religioso lo hace por sus convicciones religiosas; casi un cuarto por tradición y un $15 \%$ por la ceremonia, motivos que están más presentes entre los que no se identifican 
con alguna religión (Tabla 10). Esto se diferencia de otras ciudades como Madrid, donde la principal razón para contraer un matrimonio religioso es por tradición y sólo en segundo lugar se mencionan las convicciones religiosas (Meil, 2005). Esto estaría indicando que aun cuando disminuya el compromiso religioso de parte de los jóvenes, seguramente se seguirá produciendo matrimonio religioso.

El principal motivo para casarse tiene todavía relación con el ideal de amor romántico, especialmente entre los jóvenes de clase alta (Tabla 11). En segundo lugar de importancia se menciona la adquisición de una vivienda propia, lo que aparece como una demanda más explícita entre los jóvenes de clase media y baja, seguramente porque aquí hay más problemas económicos como para establecerse de manera independiente a sus padres. En tercer lugar se menciona la necesidad de sentirse más seguros en su relación de pareja, sin distinción por nivel socioeconómico. En cuarto lugar se asocia el matrimonio con la presencia de hijos:

Me parece bien convivir, pero depende de qué quiero [...] si quiero hijos, preferiría casarme, por lo menos [...] o sea, se exige el civil para lo religioso [...] pero yo soy bien católica y creo en el matrimonio en condiciones eclesiásticas, y me gustaría casarme por esa parte. ${ }^{10}$

Yo me iría a vivir con mi pololo, pero si quedo embarazada [...] él quiere casarse. ${ }^{11}$

Yo creo en la convivencia, pero no en la convivencia mezclada con hijos digamos [...] una cosa es convivencia y la otra es convivencia ya con hijos [...] yo creo que con hijos, sí debería haber un vínculo mayor que yo lo llamo en este caso matrimonio [porque] alguien te tiene que proteger [...] porque qué pasa, si el Estado no dicta leyes, entonces cada uno va a decir bueno yo lo voy a hacer porque le quiero a mi hijo [...] En ese sentido yo no comparto la convivencia mezclada con hijos. ${ }^{12}$

De los datos anteriores se desprende que el matrimonio todavía tiene importancia para los jóvenes, tanto desde un punto de vista instrumental (en tanto brinda seguridad) como normativo (tradición). La relación entre seguridad y matrimonio es aceptada por más de la mitad de

10 Entrevista mujer, soltera, de nivel socioeconómico medio alto.

11 Grupo focal, liceo, de nivel socioeconómico medio bajo.

12 Grupo focal, universidad, de nivel socioeconómico medio alto. 
los jóvenes, especialmente por los que tienen entre 15 y 18 años (Tabla 12). La percepción del matrimonio como un mero contrato legal que no aporta mucho a la pareja no es mayoritaria, lo que es coherente con lo anteriormente señalado.

Para mí casarse ante la Iglesia es muy importante y sí también creo que el compromiso [es importante] y también un poco darle seguridad a los hijos, también me gustaría que se formalizara ante el Estado. ${ }^{13}$

Me gustaría casarme para hacer las cosas como se deben, por el compromiso que significa. ${ }^{14}$

Yo sí me casaría pero no tengo argumentos para eso, es una cosa como de cultura, así, por él que creciera en una familia de bienestar como legalizada, pero no sé por qué, no hay como un argumento digamos, es como una cosa de tradición yo creo. ${ }^{15}$

Muy sinceramente creo en el matrimonio sí; soy católico, creo en el matrimonio más que por una cosa fundamentalista, una cosa de compromiso y eso digamos, y creo que el matrimonio es el que sustenta a la familia digamos [...] yo creo que a lo que apunta el matrimonio es a la familia digamos, y en ese sentido es a donde tenemos que llegar no importa por dónde digamos. No importa si al final convivís, vivís, matrimonio civil, religioso, sino que se pueda armar una familia y que esa familia sea indisoluble digamos. ${ }^{16}$

Estas ideas acerca del matrimonio se relacionan con las decisiones que han tomado los jóvenes en cuanto a sus propias vidas. Así, los jóvenes que perciben el matrimonio desde un punto de vista más instrumental (ventajas económicas, cuando hayan adquirido una vivienda, seguridad) tienden a convivir más que el resto; por otra parte, los que asocian el matrimonio con lo normativo (por tradición, porque es socialmente aceptable o cuando se tienen hijos) tienden a estar más casados. Estas relaciones continúan siendo significativas al controlar por edad.

Respecto de las opiniones de los jóvenes sobre la cohabitación, ésta es ampliamente aceptada, ya sea en términos de que directamente les parezca bien (48\%) o bien que se planteen indiferentes (45\%); sólo

13 Grupo focal, universidad, de nivel socioeconómico medio alto.

14 Entrevista mujer, conviviente, de nivel socioeconómico medio bajo.

15 Grupo focal, universidad, de nivel socioeconómico medio alto.

16 Grupo focal, universidad, de nivel socioeconómico medio alto. 
a una minoría le parece mal. Estas opiniones son coherentes con las expectativas que tienen los jóvenes acerca de casarse o convivir, en el sentido que a los que les parece mal mayoritariamente quieren casarse sin convivir antes. ${ }^{17}$

La cohabitación tiene un doble significado para los jóvenes: por un lado, es concebida como una etapa de prueba, que puede conducir finalmente a matrimonios más estables. Esta versión es altamente valorada por los jóvenes y, como se ha señalado anteriormente, una importante proporción de los jóvenes lo tiene proyectado hacer (Tabla 9):

Conviviría primero y de ahí me casaría para conocer más a la persona [...] me gustaría conocer más a una persona, porque hay gente que cambia al momento de casarse, entonces al conviviendo uno podría saber si seguiría igual y ahí mismo casarse después. ${ }^{18}$

[Convivir] no es al lote, es conocer más a la persona [...] porque de repente te puedes casar con un gallo [...] y te muestra doble careta y no te das cuenta y te casas toda maravillosa [...] y después te das cuenta que el gallo no era lo que pensabas [...] Entonces convivir para conocerlo más [...] cambia mucho al vivir con una persona que pololear con una persona y después casarte [...] pero al convivir sabes cómo se porta, cuáles son sus defectos, las virtudes. ${ }^{19}$

Pero igual creo que sería interesante el hecho de convivir antes de casarte [...] pero también como que yo tengo un susto personal, tal vez por experiencias personales digamos, al tema del divorcio. No tanto por problemas de moral [...] sino más bien por el hecho de que mi mamá y mi papá son separados [...] en ese sentido igual podría ser viable una cosa así, digamos el hecho de convivir antes. Pero [...] yo siempre me veo casado a largo plazo [...] y creo que es bueno casarse en el sentido de que uno admite un compromiso serio y eso ya en el plano matrimonial. ${ }^{20}$

Me gustaría convivir antes de casarme, porque yo encuentro que es fundamental, porque puede ser súper lindo, y no puedo estar todo el día, pero no es lo mismo ya a vivir con él [...] por eso me gustaría experimentar una convivencia, porque resulta que $[\ldots]$ hasta que pueda dejar

17 Elaboración propia a partir de Encuesta sobre Jóvenes y Familia.

18 Grupo focal, liceo, nivel socioeconómico medio bajo.

19 Grupo focal, colegio, nivel socioeconómico medio alto.

20 Grupo focal, colegio, nivel socioeconómico medio alto. 
el calcetín tirado en el living me puede molestar entonces no sé como que, es como mejor probar, encuentro yo. Yo creo harto en el matrimonio pero igual me gustaría poder convivir antes de casarme [...] Encuentro que así puedes conocer en un grado mayor a la persona y saber con quién te estás casando. ${ }^{21}$

Es que convivir es como ganar experiencia y después cuando se casan es el resultado de esa experiencia. ${ }^{22}$

Por otra parte, la cohabitación puede constituirse en una experiencia de más largo plazo, como alternativa al matrimonio, la cual, como se ha señalado anteriormente, está menos presente en el horizonte vital de los jóvenes (Tabla 9). Este tipo de experiencia puede ser producto de una decisión razonada de los jóvenes, especialmente entre aquellos que valoran poco el matrimonio y prefieren la convivencia en contraposición al matrimonio. La escasa valoración del matrimonio se relaciona, por una parte, con que éste se fundamenta sólo en la relación amorosa entre los miembros de la pareja. Si esto es así, el compromiso y el lazo afectivo entre las personas no requiere necesariamente de la firma del matrimonio.

Yo conviviría feliz, no me importa. O sea, no tengo problemas valóricos ni nada [...] para mí el matrimonio no equivale al peso que tiene el compromiso que admite uno con otra persona. Tengo por ejemplo mamás de amigas que no son casadas, pero que tienen el compromiso de familias, y familias de más de 35 años, hijos de 20 y 18 años y no están casados por el civil porque creo que el compromiso va por otra parte, va más por la persona. Entonces yo sí creo que uno puede convivir, sin ningún rollo. ${ }^{23}$

Por otra parte, hay una considerable cantidad de jóvenes que cree que el matrimonio limita la libertad de los cónyuges en comparación con los que conviven (42\%), especialmente entre los más jóvenes (Tabla 12). En este sentido, hay un cierto temor a casarse y a que la relación de pareja pueda cambiar como producto del matrimonio.

Convivir no sé, como que la relación tiene más vida, en cambio cuando la gente se casa como que se vuelve fome, en cambio a la convivencia

21 Grupo focal, instituto profesional, nivel socioeconómico medio.

22 Grupo focal, colegio, nivel socioeconómico medio alto.

23 Grupo focal, universidad, nivel socioeconómico medio alto. 
yo le encuentro más ventajas, pero tampoco no rechazo la idea de casarme, pero para mí es un contrato. ${ }^{24}$

Sin embargo, cabe tener en consideración que no toda experiencia de cohabitación responde necesariamente a una decisión razonada o a un deseo real de los jóvenes. En algunos casos el estado de cohabitación es una situación en cierta medida impuesta a los jóvenes, por ejemplo, cuando se tienen hijos no deseados y mientras no se tengan los recursos económicos como para independizarse de los padres. De hecho, sólo a un 30\% de los jóvenes convivientes les gustaría convivir con su pareja sin casarse más adelante; a un $68 \%$ les gustaría casarse en algún momento. ${ }^{25} 26$

Tabla 9

Expectativas de vida en pareja según edad y nivel socioeconómico (jóvenes no casados) (en porcentajes)

\begin{tabular}{|l|c|c|c|c|c|c|c|}
\hline & \multicolumn{3}{|c|}{ Edad } & \multicolumn{3}{c|}{ Nivel socioeconómico } & Total \\
\cline { 2 - 7 } & $\mathbf{1 5 - 1 8}$ & $\mathbf{1 9 - 2 4}$ & $\mathbf{2 5 - 3 0}$ & Bajo & Medio & Alto & \\
\hline $\begin{array}{l}\text { Le gustaría casarse, pero } \\
\text { antes preferiría convivir }\end{array}$ & 19,3 & $28,5^{1}$ & $36,3^{1}$ & 31,9 & 26,6 & 24,3 & 27,9 \\
\hline $\begin{array}{l}\text { Le gustaría casarse algún } \\
\text { día, pero sin convivir antes }\end{array}$ & 17,7 & 12,4 & 15,0 & $11,2^{3}$ & 14,8 & 19,4 & 14,8 \\
\hline $\begin{array}{l}\text { Le gustaría vivir solo/a un } \\
\text { tiempo y más tarde casarse }\end{array}$ & $34,6^{2}$ & $29,2^{2}$ & 17,8 & $22,7^{3}$ & 28,0 & 33,7 & 27,6 \\
\hline $\begin{array}{l}\text { Le gustaría vivir solo/a un } \\
\text { tiempo y más tarde convivir }\end{array}$ & 12,3 & 8,6 & $5,1^{1}$ & 6,5 & 7,9 & 12,6 & 8,7 \\
\hline $\begin{array}{l}\text { Le gustaría convivir con su } \\
\text { pareja, pero sin casarse }\end{array}$ & 8,4 & 13,1 & $17,1^{1}$ & $15,4^{3}$ & $15,2^{3}$ & 6,5 & 12,8 \\
\hline Le gustaría vivir solo/a & 7,7 & 8,2 & 8,8 & $12,2^{3}$ & 7,5 & 3,6 & 8,2 \\
\hline Total & $\mathrm{N}=305$ & $\mathrm{~N}=411$ & $\mathrm{~N}=288$ & $\mathrm{~N}=383$ & $\mathrm{~N}=337$ & $\mathrm{~N}=284$ & $\mathrm{~N}=1003$ \\
\hline
\end{tabular}

1 Prueba de comparación de proporciones respecto de «15-18 años» es significativa al nivel de 0,05 (prueba bilateral).

2 Prueba de comparación de proporciones respecto de «25-30 años» es significativa al nivel de 0,05 (prueba bilateral).

3 Prueba de comparación de proporciones respecto de «nivel alto» es significativa al nivel de 0,05 (prueba bilateral).

24 Grupo focal, liceo, nivel socioeconómico medio bajo.

25 Elaboración propia a partir de Encuesta sobre Jóvenes y Familia.

26 El resto preferiría vivir solo. 
Tabla 10

Expectativas de vida en pareja y razones para contraer matrimonio religioso según identificación religiosa (en porcentajes)

\begin{tabular}{|c|c|c|c|c|c|}
\hline & \multicolumn{3}{|c|}{ Identificación con religión } & \multirow[t]{2}{*}{ Total } \\
\hline & & Católica & Otra & Ninguna & \\
\hline \multirow{5}{*}{$\begin{array}{l}\text { Le gustaría casarse o convivir } \\
\text { (base: no están casados) }\end{array}$} & Casarse sin convivir & $45,9^{1}$ & $47,6^{1}$ & 29,6 & 42,3 \\
\hline & Convivir & $17,3^{1}$ & $17,9^{1}$ & 34,4 & 21,5 \\
\hline & \begin{tabular}{|l|} 
Convivir y casarse \\
\end{tabular} & 29,7 & 22,6 & 28,1 & 27,9 \\
\hline & Le gustaría vivir solo & 7,1 & 11,9 & 7,9 & 8,2 \\
\hline & Total & $\mathrm{N}=582$ & $\mathrm{~N}=189$ & $\mathrm{~N}=230$ & $\mathrm{~N}=1003$ \\
\hline \multirow{5}{*}{$\begin{array}{l}\text { Por qué le gustaría/gustó } \\
\text { tener un matrimonio religioso } \\
\text { (base: le gustaría o se casó } \\
\text { por matrimonio religioso) }\end{array}$} & Por tradición & $21,7^{1}$ & $18,8^{1}$ & 45,9 & 23,2 \\
\hline & $\begin{array}{l}\text { Porque es lo que su } \\
\text { familia espera }\end{array}$ & 6,3 & 7,8 & 5,9 & 6,6 \\
\hline & $\begin{array}{l}\text { Porque le gusta la } \\
\text { ceremonia }\end{array}$ & $14,4^{1}$ & $10,4^{1}$ & 29,2 & 14,9 \\
\hline & $\begin{array}{l}\text { Por sus convicciones } \\
\text { religiosas }\end{array}$ & $57,6^{1}$ & $63,0^{1}$ & 18,9 & 55,3 \\
\hline & Total & $\mathrm{N}=479$ & $\mathrm{~N}=136$ & $\mathrm{~N}=59$ & $\mathrm{~N}=674$ \\
\hline
\end{tabular}

Prueba de comparación de proporciones respecto de «ninguna religión» es significativa al nivel de 0,05 (prueba bilateral).

Tabla 11

Motivos para decidir casarse según nivel socioeconómico (en porcentajes)

\begin{tabular}{|c|c|c|c|c|}
\hline \multirow{2}{*}{$\begin{array}{l}\text { Motivos para decidir casarse } \\
\text { (respuesta múltiple, hasta } 3 \text { menciones) }\end{array}$} & \multicolumn{3}{|c|}{ Nivel socioeconómico } & \multirow[t]{2}{*}{ Total } \\
\hline & Bajo & Medio & Alto & \\
\hline Cuando se sienta totalmente seguro del amor entre la pareja & 70,6 & 77,7 & 87,2 & 77,5 \\
\hline Cuando hayan adquirido una vivienda propia & 48,4 & 50,0 & 33,1 & 44,7 \\
\hline Para sentirse más seguro/a en su relación de pareja & 40,9 & 43,9 & 39,1 & 41,4 \\
\hline Cuando quieran tener hijos con su pareja & 25,0 & 34,5 & 41,2 & 32,6 \\
\hline Es lo que la familia espera & 24,9 & 22,0 & 15,7 & 21,4 \\
\hline Socialmente es mejor considerado casarse antes que convivir & 20,8 & 16,0 & 9,9 & 16,2 \\
\hline Para aprovechar las ventajas económicas del matrimonio & 13,5 & 12,3 & 7,7 & 11,5 \\
\hline En caso de embarazo & 13,1 & 10,0 & 8,6 & 10,8 \\
\hline No le interesa casarse & 2,8 & 1,6 & 2,2 & 2,3 \\
\hline Casos & 469 & 395 & 332 & 1196 \\
\hline Respuestas & 1220 & 1058 & 811 & 3089 \\
\hline
\end{tabular}


Tabla 12

Ideas sobre el matrimonio según grupos de edad (en porcentajes)

\begin{tabular}{|l|c|c|c|c|}
\hline & \multicolumn{3}{|c|}{ Edad } & \multirow{2}{*}{ Total } \\
\cline { 2 - 4 } & $\mathbf{1 5 - 1 8}$ & $\mathbf{1 9 - 2 4}$ & $\mathbf{2 5 - 3 0}$ & \\
\hline Acuerdo con que casarse da más seguridad que convivir & 67,6 & $49,8^{1}$ & $52,9^{1}$ & 55,5 \\
\hline $\begin{array}{l}\text { Acuerdo con que los hijos crecerán más seguros si sus } \\
\text { padres están casados }\end{array}$ & 70,6 & $59,0^{1}$ & $53,7^{1}$ & 59,9 \\
\hline $\begin{array}{l}\text { Acuerdo con que los que se casan tienen menos libertad } \\
\text { que los que conviven sin casarse }\end{array}$ & 54,6 & $38,7^{1}$ & $37,8^{1}$ & 42,3 \\
\hline $\begin{array}{l}\text { Acuerdo con que un matrimonio es un contrato legal } \\
\text { que no aporta mucho a la pareja }\end{array}$ & 41,4 & 44,4 & 40,2 & 42,1 \\
\hline & $\mathrm{N}=301$ & $\mathrm{~N}=439$ & $\mathrm{~N}=452$ & $\mathrm{~N}=1192$ \\
\hline
\end{tabular}

Prueba de comparación de proporciones respecto de «15-18» es significativa al nivel de 0,05 (prueba bilateral).

2 Diferencias respecto a edad no son estadísticamente significativas.

\section{CONCLUSIONES}

En conclusión, se ha podido constatar que la familia resulta muy importante en los horizontes vitales de los jóvenes y la mayoría cree que va a estar casado y con hijos en el futuro.

La idea de familia se asocia especialmente a la presencia de hijos. La constitución de una pareja en matrimonio tiene importancia secundaria, prácticamente similar a convivir sin casarse en el caso que se tengan hijos. Pero cuando no existen hijos ni matrimonio, en general los jóvenes tienden a no considerar estas situaciones como familias.

Estos datos son consistentes con que la mayoría de los jóvenes desea tener hijos, aunque simplemente para ser felices en la vida antes que como un compromiso con la sociedad o la religión, o por motivos instrumentales, por ejemplo, tener alguien que los cuide en la vejez. Si bien en Chile ha caído la fecundidad, ésta todavía se mantiene por sobre los dos hijos por mujer. Las mujeres chilenas están teniendo hijos en su gran mayoría entre 2 ó 3.

La convivencia sin matrimonio es bastante aceptada, pero esto no significa que los jóvenes esperen vivenciarla en igual magnitud. Una amplia mayoría de los jóvenes se ve todavía como casados en el futuro, especialmente a través de un matrimonio religioso. La convivencia como estado permanente sólo es deseable en la vida personal

de algunos jóvenes, aunque la cohabitación premarital seguramente 
seguirá siendo una realidad cada vez más probable. No está tan claro en qué momento desean casarse los jóvenes, aunque al parecer los hijos no están entre los motivos más importantes; sólo un tercio de los jóvenes menciona tener hijos como una condición para casarse; adicionalmente, se ha podido constatar que la maternidad adolescente cada vez conduce menos al matrimonio.

El matrimonio sigue estando presente en el horizonte vital de la mayoría de los jóvenes, quienes en general se ven en el futuro como casados. Sin embargo, el modo de entrada al matrimonio ha cambiado, donde la convivencia premarital es altamente valorada. El fundamento primero del matrimonio es la relación de amor de una pareja, la que puede redundar más tarde en una relación inestable, ya que si el modo de entrada al matrimonio es principalmente afectivo, la desaparición del amor de pareja puede conducir a la disolución del matrimonio, lo que es consistente con la amplia aceptación del divorcio de parte de los jóvenes. Incluso una porción de éstos se concibe a sí mismo como separado en 25 años más. Esto no depende tanto de la situación conyugal que han experimentado los padres de estos jóvenes, sino que más bien de su entorno socioeconómico. Al parecer, el vivenciar situaciones de precariedad económica se relaciona con mayor inestabilidad de las relaciones de pareja. En este sentido, el trabajo de la mujer, que se ha demostrado se asocia con un mejoramiento de la condición económica de los hogares, podría contribuir a una mayor estabilidad de las relaciones de pareja.

SANTIAGO (CHILE), MAYO 2006

RECIBIDO: MAYO 2006

ACEPTADO: JULIO 2006

\section{REFERENCIAS BIBLIOGRÁFICAS}

DESUC (1998): Encuesta nacional de familia. Santiago: DESUC.

GubBINS, V., F. BROWNE y A. BAGNARA (2004): «Familia: innovaciones y desafíos. Las familias chilenas es la década 1992-2002». En Cómo ha cambiado la vida de los chilenos... Análisis comparativo de las condiciones de vida en los hogares con menor bienestar socioeconómico (censos 1992-2002). Santiago: INE.

HEATON, T. (2002): «Factors contributing to increasing marital stability in the United States». Journal of Family Issues, Vol. 23, No3.

HERRERA, M. S. (2002): «Individualización social y cambios demográfico- 
familiares: ¿hacia una segunda transición demográfica?». Madrid: Departamento de Sociología, Universidad Autónoma de Madrid.

HERRERA, S. y E. VALENZUELA (2006): «Matrimonios, separaciones y convivencias». En VALENZUELA, Tironi y SCUlly (ed.): El eslabón perdido. Familia, modernización y bienestar en Chile. Santiago: Taurus.

INE (2003): Anuario de estadísticas vitales 2001. Santiago: INE. (1999): Estadísticas de Chile en el siglo XX. Santiago: INE.

IsSP (1994). International Social Survey Programme.

Kiernan, K., y G. Mueller (1990): «Who divorces?». En SuSAn McRaE (ed.): Changing Britain. Families and Households in the 1990s. Oxford: Oxford University Press.

MEIL, G. (2005): «La pareja en los proyectos vitales de los jóvenes: deseos y realidades». Revista de Estudios de Juventud $\mathrm{N}^{\circ} 67$. Madrid: INJUVE.

Orbuch, T., J. VEROFF, H. HASSAN y J. HORROCKS (2002): «Who will divorce: a 14-year longitudinal study of back couples and white couples». Journal of Social and Personal Relationships, Vol. 19, №2.

United NATIONS (varios años): Demographic Yearbook. New York: United Nations.

VAlenZuela, E., y S. HerRera (2003): «Movilidad residencial y movilidad social». En Cuánto y cómo cambiamos los chilenos. Balance de una década. Santiago: INE.

WELLINGS, K., y J. WADSWORTH (1990): «Family influences on teenage fertility». En SUSAN MCRAE (ed.): Changing Britain. Families and Households in the 1990s. Oxford: Oxford University Press.

WOLFINGER, N. (2000): «Beyond the intergenerational transmission of divorce». Journal of Family Issues, Vol. 21, $\mathrm{N}^{\circ} 8$. 